\title{
Investigation of Anti-Coronavirus, Anti-HCV, Nucleotide Inhibitors, and Bioactive Molecules efficacy Against RNA-directed RNA polymerase of Nipah Virus: Molecular Docking Study
}

Peter T. Habib ( $\sim$ p.habib911@gmail.com )

Colors Medical Laboratories

\section{Research Article}

Keywords: Molecular Docking, Nipah Virus, Antiviral Molecules and Drug

Posted Date: March 4th, 2021

DOI: https://doi.org/10.21203/rs.3.rs-294115/v2

License: (9) This work is licensed under a Creative Commons Attribution 4.0 International License. Read Full License 


\section{Abstract}

The infections with the Nipah virus (NiV) are highly infectious and may lead to severe febrile encephalitis. High rates of mortality in southeastern Asia including Bengal, Malaysia, Papua New Guinea, Vietnam, Cambodia, Indonesia, Madagascar, the Philippines, Thailand, and India have been reported in NiV outbreaks. Considering the high risk of an epidemic, NiV was declared a priority pathogen by the World Health Organization (WHO). However, for the treatment of this infection, there is no effective therapy or approved FDA medicines. RNA-dependent polymerase RNA (RdRp) plays an important role in viral replication among the nine well-known proteins of NiV. Therefore, fourteen antiviral molecules have been computerized for NiV RNA-dependent RNA polymerase and demonstrated a potential inhibition effect against coronavirus (NiV-RdRp). A multi-step molecular docking process, followed by extensive analyzes of molecular binding interactions, binding energy estimates, synthetic accessibility assessments, and toxicity tests. Analysis reveals that Uprifosbuvir is the most suitable inhibitor for RdRp of Nipah Virus regarding the binding affinity and binding in the target cavity. Although, such studies need clinical confirmation.

\section{Introduction}

Nipah virus (NiV) was originally identified during an outbreak in Malaysia in 1999, was associated with the Hendra virus in the Paramyxoviridae group [1]. Since then, sporadic NiV outbreak outbreaks have been reported in several countries, including the latest in Indian Kerala [2]. NiV is endemic, highly pathogenic, and can infect most species of mammals. Henipavirus natural reservoirs seem to be Pteropus-type fruit bats [3]. However, NiV has a wide range of hosts, such as humans [4] which lead to serious neurological, respiratory, and cardiovascular illnesses. Hosts include horses, dogs, dogs, pigs, cats, and hamsters. Since NiV can be passed from zoonotic to human routes, the risk for outbreaks is very high [5]. Particularly, The NiV outbreaks are the most prominent for human-to-human transmission. About 600 cases of NiV human infections were reported between 1998 and 2015, where the fatality rate in Malaysia was $38 \%$ and $43-100 \%$ in India and Bangladesh [2].

Due to its persistent pathogenicity, high mortality, and lack of effective therapeutics against its infection, $\mathrm{NiV}$ is a biosafety level 4 (BSL4) pathogen [6]. Some efforts have been made to manage NiV in the search for small molecular therapy for various structural proteins [7], [8]. Favipiravir has been shown to combat viral reproduction and transcription in NiV and $\mathrm{HeV}$ in a recent in vitro study; [9]. Also reported was R1479 (4'-azidocytidine) to inhibit various family members of Paramyxoviridae [10]. Nevertheless, no approved medicines are yet available for efficient human usage despite these efforts to develop such inhibitors. While Ribavirin is not an established NiV treatment, it is especially used in a state of emergency as a firstline treatment strategy for acute NiV encephalitis [11]; It does however have different side effects during NiV therapy, such as nausea, vomiting, and convulsions [12]. Known for treating NiV, small molecules inhibit host proteins such as Interferon Regulatory Factor 3 and RIG-Ilike receptors which contribute innate immune system [13]. No approved therapeutics are available to efficiently use in humans despite these efforts to develop such inhibitors. Therefore, the definition of potential NiV inhibitors is an eminent 
requirement. Various efforts were made to manage NiV infection by looking for small molecular therapy for various structural proteins [7], [8], [14], [15].

The structure of the NiV virus is a parallel, long, coiled-coil, tetrameric with a small helical cap as tethers of the viral polymerase to nucleocapsid and multimeric phosphoprotein. Nipah virus has a singlestranded, negative-sense RNA genome that is encapsulated by the nucleoprotein $(\mathrm{N})$ and transcribed and replicated by the polymerase protein $(L)$. Hence molecular inhibition of RNA polymerase is one of the prime strategies for treating NiV infection. Also, as an effective strategy to interrupt virus adhesion with the host, the inferences regarding inhibition of interactions between RNA polymerase and RNA, which provide the structural basis for screening antiviral therapeutic inhibitors against NiV.

The most reliable and widely used approach in the early stages of drug discovery is structure-based molecular docking [16]; However, information on their potential side effects leading to almost 30 percent failure of the drug applicants due to toxicity or clinical safety problems is not generally taken into account [17]. The drug's off-target toxicity and chemical structure that leads to its non-optimal efficacy [18] is predicted to cause these side effects [19]. There is therefore no understanding of the assessment of drug toxicity at the level of molecular interaction relying only on this method. Different approaches should be used to control any possible pandemic of Nipah virus such as using machine learning for detection of different strains [20], [21], early detection of respiratory syndromes[22], and developing new drugs or scanning for possible molecule that may target and inhibit viral life cycle [22].

This is linked to the observation that interactions between drugs and their direct target frequently lead to differential expressions of downstream target regulated genes (Noh, Shoemaker, \& Gunawan, 2018), which cause potential activation, repression, or dysregulation of downstream pathways. Here we introduce a molecular docking study for fourteen possible molecules and drug may act against RNAdependent RNA polymerase of Nipah virus. Some previously efforts developed vaccine based on analysis of the whole proteome[23].

\section{Materials And Methods}

The method, software, and result analysis was imported from Habib's work[15].

\section{Ligands Retrieving and preparation}

The chemical structures of selected ligands were retrieved from PubChem

(https://pubchem.ncbi.nlm.nih.gov/), DrugBank (https://go.drugbank.com/), and SANCDB (https://sancdb.rubi.ru.ac.za/) in Protein database (PDB) format and then converted autodock ligand in the format of .pdbqt format using openbabel.

\section{Protein Modeling, Retrieving, and Preparation}

RNA-dependent RNA Polymerase (RdRp) of the Nipah virus still has no identified structure. So, A homology model for the Nipah RdRp was built using the Swiss Model webserver (Biasini et al. , 2014). We 
used the available RdRp sequence of the Nipah virus to build a model for the unknown structure. SWISSMODEL shows RNA-directed RNA polymerase $L$ of Parainfluenza as a similar structure with $36 \%$ identity and $95 \%$ coverage, which was the best result among all templates. Analysis of protein structure was quite good where Ramachandran plot archives $84.82 \%$ in the favored region (Figure 1) and MolProbity score was 1.98 .

\section{Absorption, distribution, metabolism, excretion, and toxicity profile and druggability}

Pharmacokinetic parameters of a compound like absorption, distribution, metabolism, excretion, and toxicity play a vital role in the drug discovery process. Hence, an online server Swiss-Adme (http://www.swissadme.ch/index.php) was used to predict the psychochemical properties of each ligand. We predicted GI absorption, BBB permeant, Pgp substrate, a CYP1A2 inhibitor, CYP2C19 inhibitor, CYP2C9 inhibitor, CYP2D6 inhibitor, a CYP3A4 inhibitor, Lipinski violations, Bioavailability Score, Leadlikeness, and Synthetic Accessibility as shown in (Figure 2).

\section{Protein-Ligand Docking}

Docking was performed using autodock4 at PyRx (https://pyrx.sourceforge.io/) 0.8 platform. After docking ten different poses of ligand molecules were obtained; the pose scoring lowest binding energy was chosen to visualize the ligand-protein interaction using Discovery studio 2019.

\section{Results}

\section{SWISS-ADME prediction of ligands}

We studied probable physiological properties of each selected ligand to cross the blood-brain barrier, human intestinal absorptivity, caco-2 permeability, as toxicity, carcinogen character, acute oral toxicity, rat acute toxicity, and fish toxicity. properties of selected ligand Among the selected ligands, Acetaminophen was having the best lowest Synthetic Accessibility which means it is easy to synthesize. Guanosine-5'triphosphate was able to pass Blood-Brain Barrier (BBB+) and not substrate or inhibitor for different Cytochromes. On the other hand, Uprifosbuvir is intestinally absorbable while it is 545.91 in MW.

\section{Identifying Ligand Binding Site}

Ligand binding site, as the term suggests, is the site where the ligand binds. To define the binding site, we added hydrogen atom to all cavities in protein that may act as the binding site and then identify the possible binding sites from the possible receptor atoms, as it is shown in (Figure 2)

\section{Ligand Protein Docking}

Setrobuvir showed the highest binding affinity with CETP via $-9.6 \mathrm{kcal} / \mathrm{mol}$ binding energy. However, the binding was not in the accurate binding site. Uprifosbuvir achieves $-7.4 \mathrm{kcal} / \mathrm{mol}$ binding energy come the six in order of binding affinity molecules but it still located within the binding cavity. Remdesivir is a 
known anti-viral drug used recently against coronavirus, which binds with $-8.1 \mathrm{kcal} / \mathrm{mol}$ but outside the ligand-binding cavity. best molecules were Guanosine-5'-triphosphate (Figure 3), Mericitabine (Figure 4), and Uprifosbuvir (Figure 5).

\section{Discussion}

The current study was designed to understand the interaction of active molecules and drugs used recently in the coronavirus pandemic in inhibiting the Nipah virus by suppressing RNA-dependent RNA polymerase. Although X-ray crystallography and nuclear magnetic resonance techniques are utilized to understand the ligand-protein interaction; there is no known structure of RdRp. To overcome this limitation, homology modeling was utilized to predict the structure of the Nipah RdRb using SWISSMODEL. The location where the ligand should bind is identified. Molecular docking was performed on fourteen molecules and drugs to assess the binding affinity. The order of ligand according to affinity was: Setrobuvir, Remdesivir, Octahydroeuclein, Sofosbuvir, Uprifosbuvir, Adenosine-5'-triphosphate, Ribavirin, Galidesivir, Guanosine-5'-triphosphate with binding affinity -9.6, -8.1, -7.8, -7.6, -7.4, -7.4, -7, -7, -6.8 respectively. Many of them were located in the RdRp cavity but, the best affinity and in target molecules were Guanosine-5'-triphosphate, Mericitabine, and Uprifosbuvir with binding affinity $-6.8,-6.5$, and -7.4 respectively. As shown in (Figure 5), Uprifosbuvir forms many and different types of bond-like: Van der Waals, Hydrogen bond, Pi-Sigma, Amide-Pi-Stacked, Carbone-Hydrogen bond, Alkyl, and Pi-Alkyl. Guanosine-5'-triphosphate is only one form bond with an attractive charge between phosphate atom and Asparagine amino acid. After docking analysis, Uprifosbuvir was able to prove that is the only ligand suitable for RdRp inhibition. However, some reports show that Uprifosbuvir has a negative effect on vaccine-drug interaction. The therapeutic efficacy of Adenovirus type 7 vaccine, Anthrax vaccine, BCG vaccine, Rubella virus vaccine, Typhoid Vaccine, and Yellow Fever Vaccine can be decreased when used in combination with Uprifosbuvir. Although, Uprifosbuvir is an approved drug for the Hepatitis $C$ virus.

\section{Conclusion}

The present study represents the possible role of anti-viral molecules as a ligand against RNA-dependent RNA polymerase. Results from acute oral toxicity, rat acute toxicity, and fish toxicity predict the molecules are non-toxic, absorbable via the human intestine, and able to pass the blood-brain barrier. All the selected ligands have scored well drug-likeness scores which affect the physicochemical property of the compound either towards pharmacological action or towards the drug development steps. The obtained Log $S$ value represents the solubility of the drug molecules which would further help in the formulation steps in the pharmaceutical industry. Molecular docking study analysis reveals the Uprifosbuvir might be a candidate RdRp inhibitor. This study should encourage further investigation of the properties of the already identified anti-viral molecules followed by a pharmacological investigation of these in-silico findings in suitable models.

\section{Declarations}




\section{Compliance with ethical standards}

Conflict of interest There are no conflicts of interest to declare.

\section{References}

[1] K. B. Chua et al., "Nipah virus: a recently emergent deadly paramyxovirus," Science, vol. 288, no. 5470, pp. 1432-1435, 2000.

[2] V. K. Chattu, R. Kumar, S. Kumary, F. Kajal, and J. K. David, "Nipah virus epidemic in southern India and emphasizing 'One Health' approach to ensure global health security," Journal of family medicine and primary care, vol. 7, no. 2, p. 275, 2018.

[3] J. G. Olson et al., "Antibodies to Nipah-like virus in bats (Pteropus lylei), Cambodia," Emerging infectious diseases, vol. 8, no. 9, p. 987, 2002.

[4] M. N. M. Nor, C. H. Gan, and B. L. Ong, "Nipah virus infection of pigs in peninsular Malaysia," Revue Scientifique et Technique-Office International des Epizooties, vol. 19, no. 1, pp. 160-165, 2000.

[5] S. P. Luby, "The pandemic potential of Nipah virus," Antiviral research, vol. 100, no. 1, pp. 38-43, 2013.

[6] A. N. Freiberg, M. N. Worthy, B. Lee, and M. R. Holbrook, "Combined chloroquine and ribavirin treatment does not prevent death in a hamster model of Nipah and Hendra virus infection," The Journal of general virology, vol. 91, no. Pt 3, p. 765, 2010.

[7] B. Tigabu et al., "A BSL-4 high-throughput screen identifies sulfonamide inhibitors of Nipah virus," Assay and drug development technologies, vol. 12, no. 3, pp. 155-161, 2014.

[8] S. Niedermeier et al., "A small-molecule inhibitor of Nipah virus envelope protein-mediated membrane fusion," Journal of medicinal chemistry, vol. 52, no. 14, pp. 4257-4265, 2009.

[9] B. E. Dawes et al., "Favipiravir (T-705) protects against Nipah virus infection in the hamster model," Scientific reports, vol. 8, no. 1, pp. 1-11, 2018.

[10] A. L. Hotard, B. He, S. T. Nichol, C. F. Spiropoulou, and M. K. Lo, "4'-Azidocytidine (R1479) inhibits henipaviruses and other paramyxoviruses with high potency," Antiviral research, vol. 144, pp. 147-152, 2017.

[11] N. J. C. Snell, “Ribavirin therapy for Nipah virus infection,” Journal of virology, vol. 78, no. 18, p. 10211, 2004.

[12] H.-T. Chong et al., "Treatment of acute Nipah encephalitis with ribavirin," Annals of Neurology: Official Journal of the American Neurological Association and the Child Neurology Society, vol. 49, no. 6, pp. 810-813, 2001. 
[13] V. Guillaume et al., "Antibody prophylaxis and therapy against Nipah virus infection in hamsters," Journal of virology, vol. 80, no. 4, pp. 1972-1978, 2006.

[14] P. T. Habib, "Vaccine Design, Adaptation, and Cloning Design for Multiple Epitope-Based Vaccine Derived From SARS-CoV-2 Surface Glycoprotein (S), Membrane Protein (M) and Envelope Protein (E): In silico approach," 2021, doi: 10.21203/rs.3.rs-241638/v1.

[15] P. T. Habib and M. Saber-Ayad, "In Silico Analysis of 716 Natural Bioactive Molecules Form Atlantic Ocean Reveals Candidate Molecule to Inhibit Spike Protein," 2021, doi: 10.21203/rs.3.rs-156623/v1.

[16] X.-Y. Meng, H.-X. Zhang, M. Mezei, and M. Cui, "Molecular docking: a powerful approach for structurebased drug discovery," Current computer-aided drug design, vol. 7, no. 2, pp. 146-157, 2011.

[17] I. Kola and J. Landis, "Can the pharmaceutical industry reduce attrition rates?," Nature reviews Drug discovery, vol. 3, no. 8, pp. 711-716, 2004.

[18] A. Bender et al., "Analysis of pharmacology data and the prediction of adverse drug reactions and offtarget effects from chemical structure," ChemMedChem: Chemistry Enabling Drug Discovery, vol. 2, no. 6, pp. 861-873, 2007.

[19] L. Brouwers, M. Iskar, G. Zeller, V. van Noort, and P. Bork, "Network neighbors of drug targets contribute to drug side-effect similarity," PloS one, vol. 6, no. 7, p. e22187, 2011.

[20] P. Habib, A. Alsamman, M. Saber-Ayad, S. H.- bioRxiv, and undefined 2020, “COVIDier: a deep-learning tool for coronaviruses genome and virulence proteins classification," biorxiv.org, Accessed: Mar. 03, 2021. [Online]. Available: https://www.biorxiv.org/content/10.1101/2020.05.03.075549v1.abstract.

[21] P. H.- bioRxiv and undefined 2020, "COVATOR: A Software for Chimeric Coronavirus Identification," biorxiv.org, Accessed: Mar. 03, 2021. [Online]. Available:

https://www.biorxiv.org/content/10.1101/2020.11.14.383075v1.abstract.

[22] P. Habib, A. Alsamman, ... S. H.-H. in, and undefined 2020, “Developing Convolutional Neural Networks-Based System for Predicting Pneumonia Using X-Radiography Image," highlightsin.org, Accessed: Mar. 03, 2021. [Online]. Available:

http://highlightsin.org/index.php/bioscience/article/view/14.

[23] P. T. Habib, "Learning from COVID-19 Pandemic: In Silico Vaccine and Cloning Design Against Nipah Virus by Studying and Analyzing the Whole Nipah Virus Proteome," 2021, doi: 10.21203/rs.3.rs269666/v1.

\section{Figures}




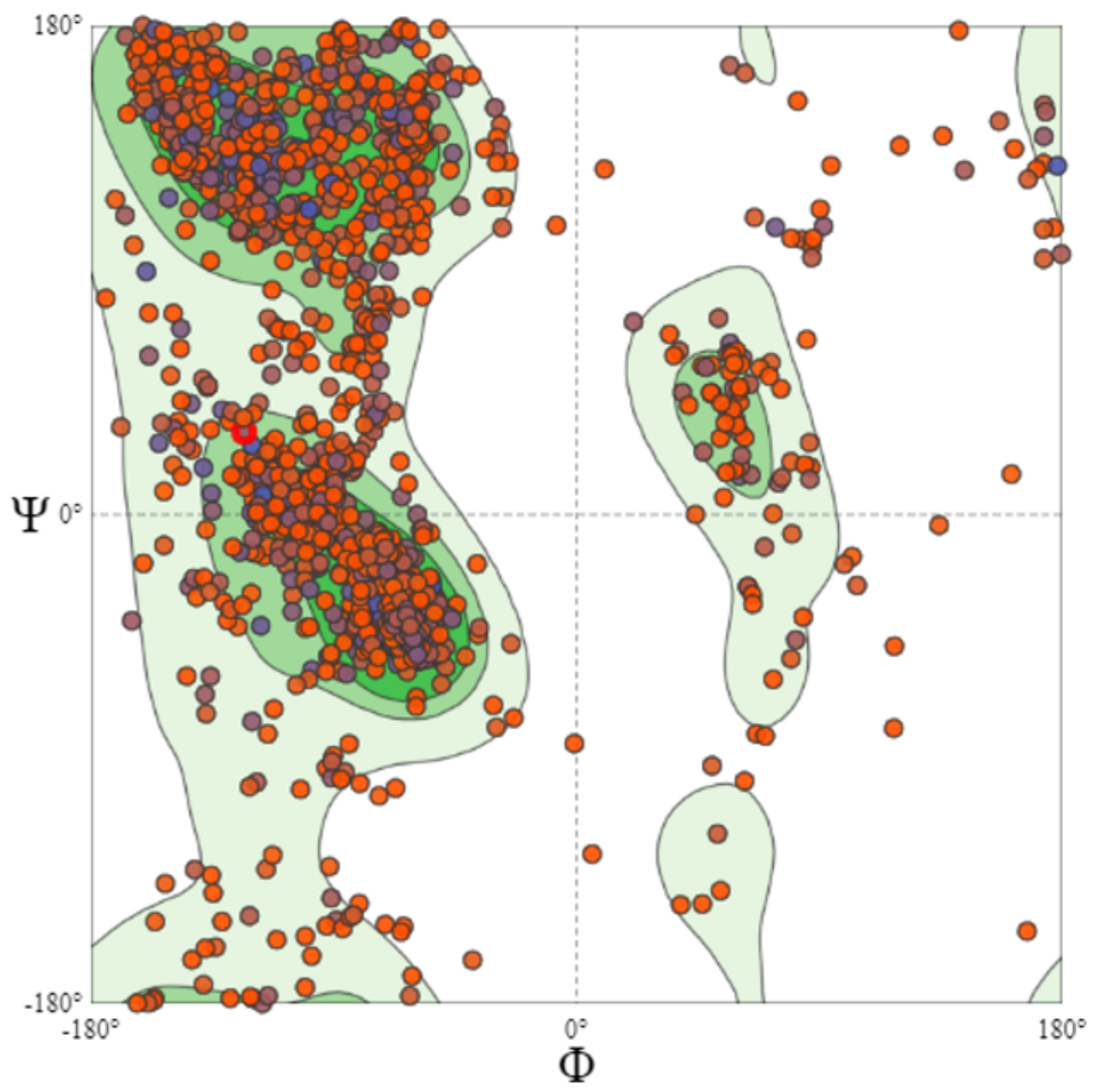

Figure 1

Ramachandran plot of RNA-dependent RNA polymerase of parainfluenza virus 


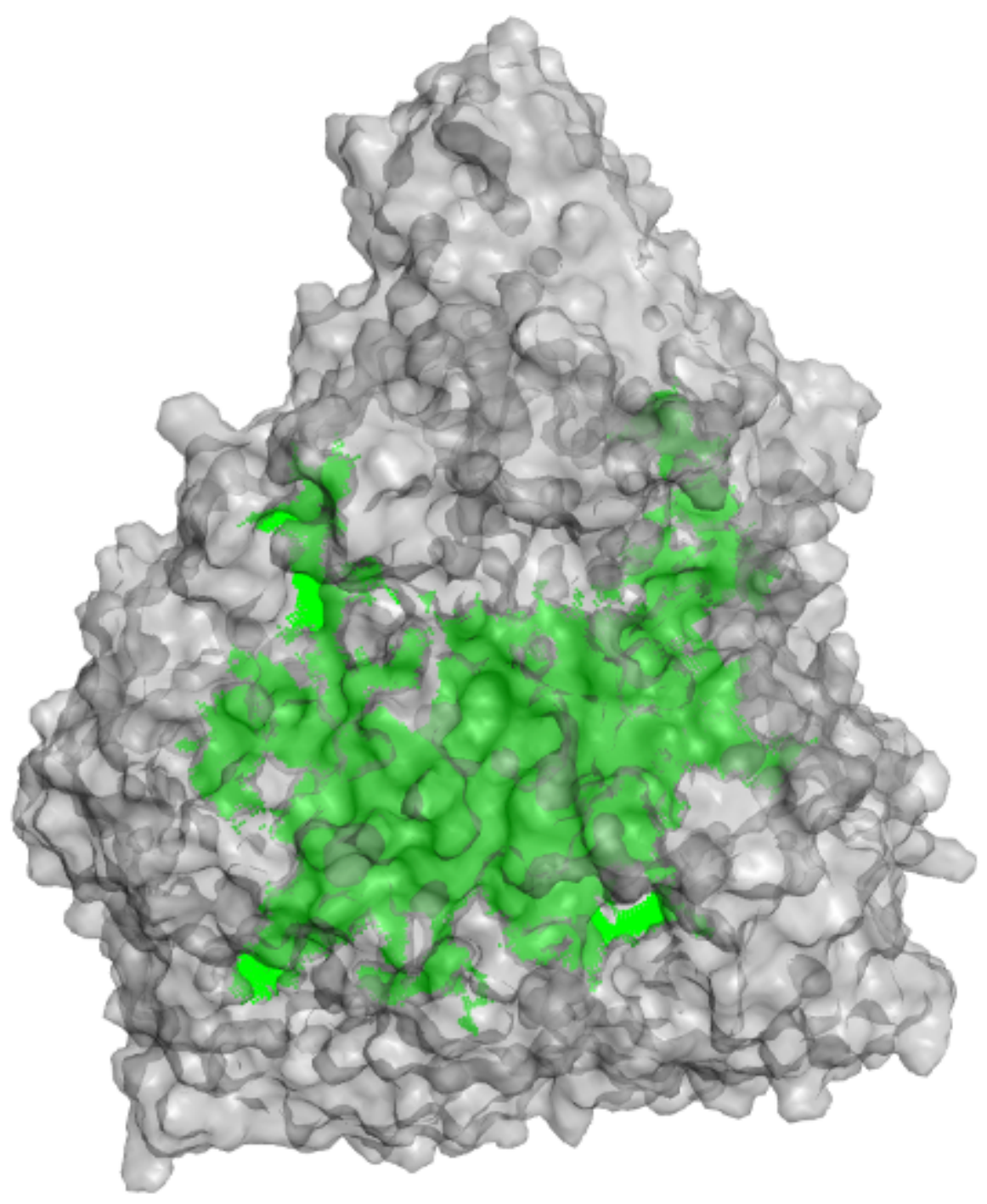

Figure 2

Predicted Binding site (green) inside the protein (Gray) cavity 

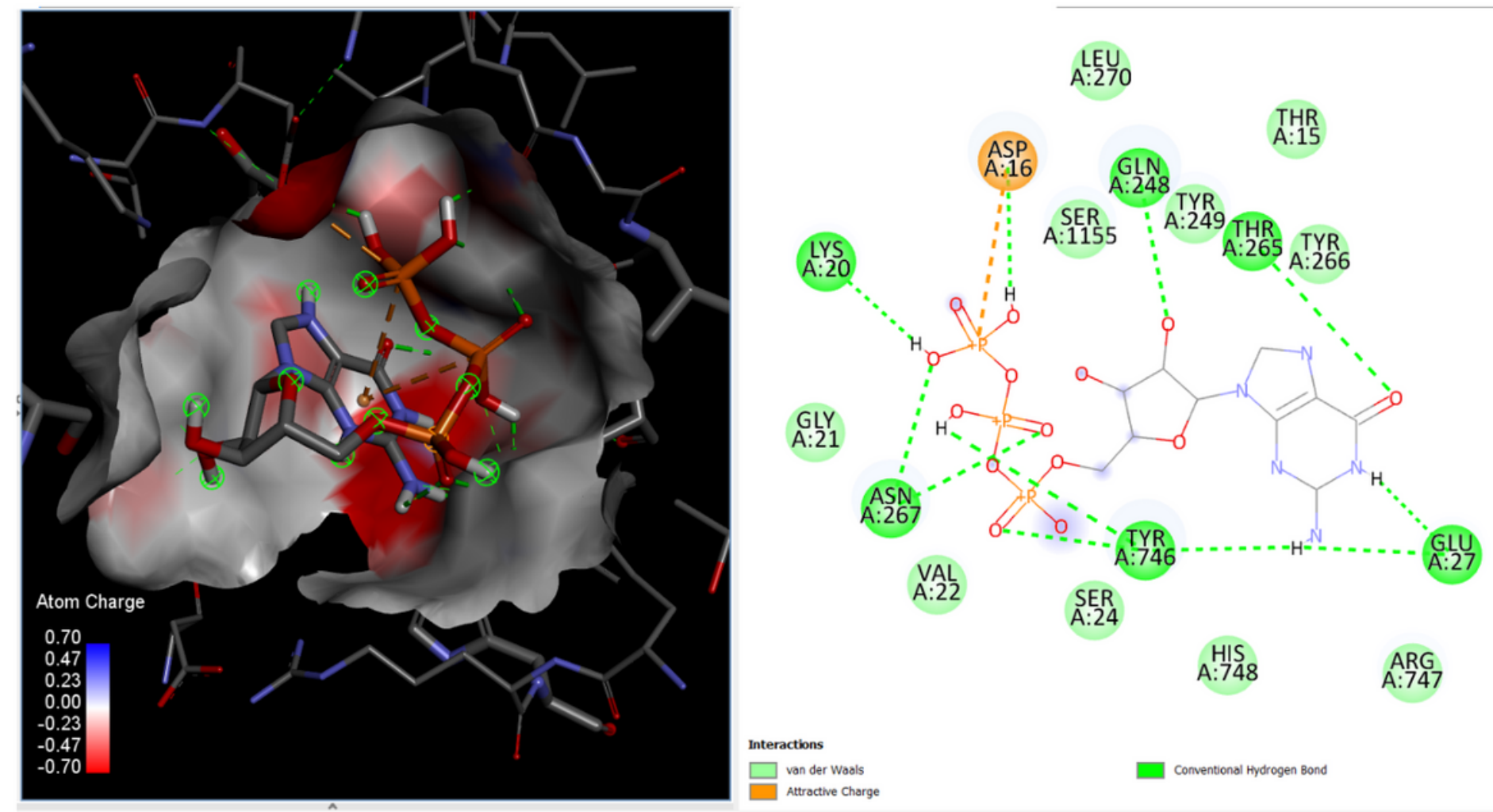

Figure 3

Guanosine-5'-triphosphate binding site (Left) and atoms interaction (Right)
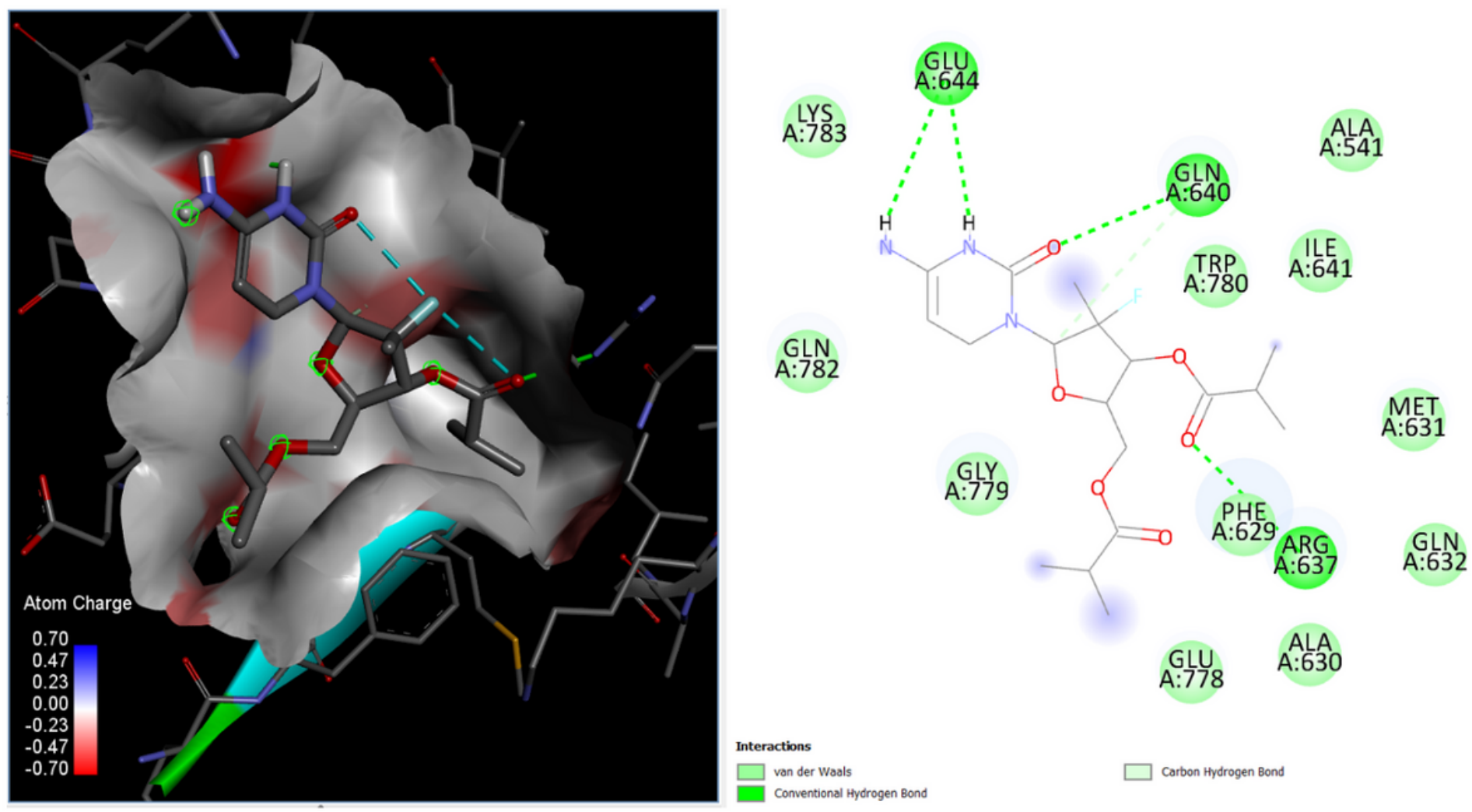

Figure 4 
Mericitabine binding site (Left) and atoms interaction (Right)
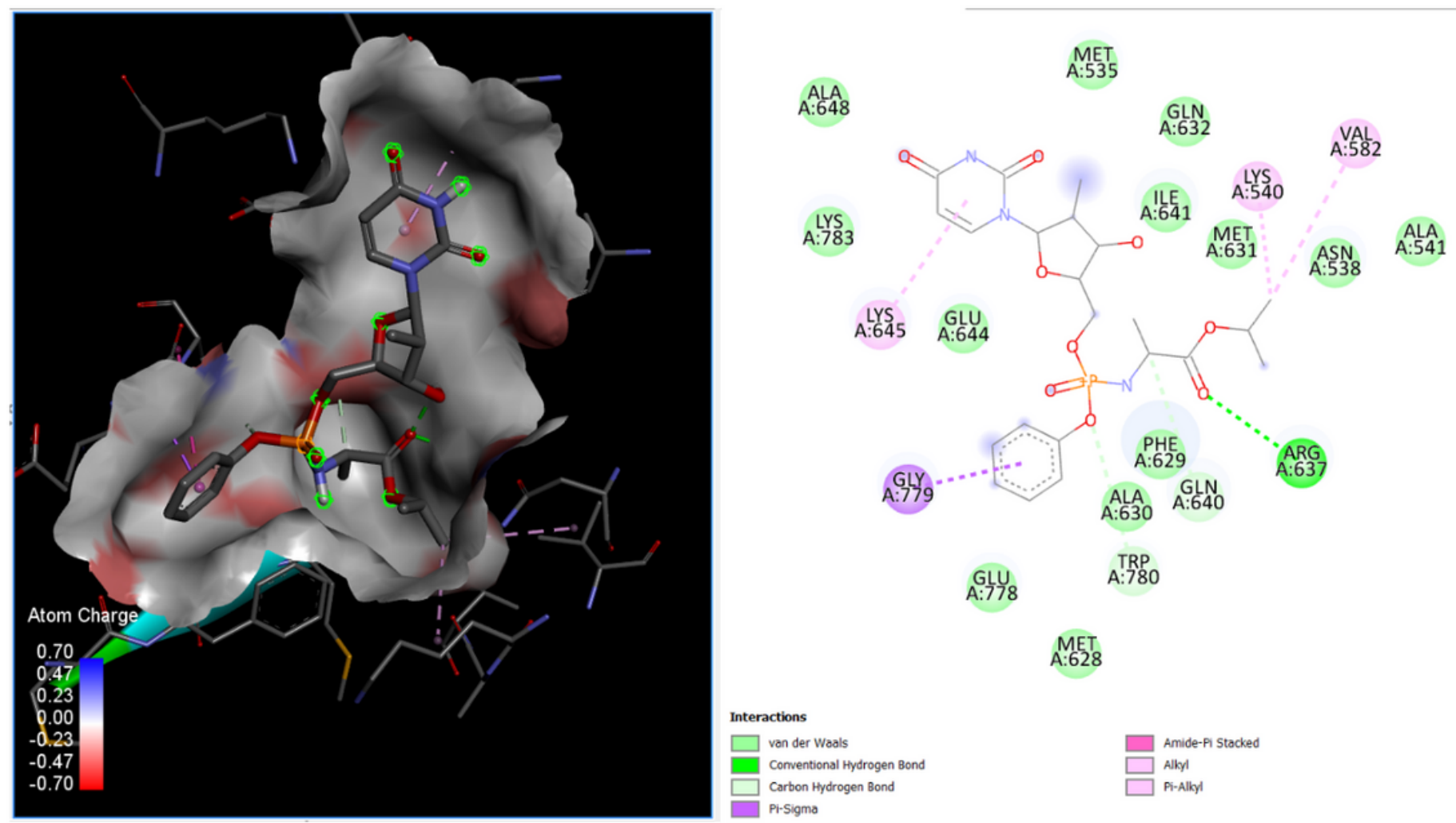

Figure 5

Uprifosbuvir binding site (Left) and atom interaction (Right) 